ReApeRs

\author{
Journal of Research in Psychology (JRP) \\ www. readersinsight.net/jrp \\ ISSN: 2664-9500 (Online) \\ ISSN: 2664-9497 (Print)
}

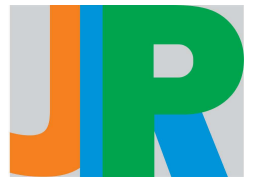

\title{
Enhancing Learning Process using Double-Loop Theory in Purchasing Process of Healthcare Organization Toward Cutting Unnecessary Costs.
}

\author{
Tiyib M. Mohmoud ${ }^{1}$, Zulkifli H. Khair ${ }^{2}$, Muhammed F. Bin Othman ${ }^{3}$ \\ ${ }^{1}$ Azman Hashim Int. Bus. School, Faculty of Management, Universiti Teknologi Malaysia 81310 Johor Bahru, Johor, Malaysia \\ Pediatric Cardiac Center of Excellence, King Abdulaziz University Hospital, Jeddah, Saudi Arabia \\ ${ }^{2}$ Faculty of Management, Universiti Teknologi Malaysia 81310 Johor Bahru, Johor, Malaysia \\ ${ }^{3}$ Faculty of Management, Universiti Teknologi Malaysia 81310 Johor Bahru, Johor, Malaysia \\ *Corresponding author: altayeb.abdullah@gmail.com, zuhaka@gmail.com
}

\begin{abstract}
The reports show that America is currently paying around $17.9 \%$ of the national annual budget on expenses of health care services with expecting to be raised of up to $19.6 \%$ in 2021 , same with other developed and developing countries. This makes alarming and pushes our look again of the way of management of the operations of health care systems, especially when the increased costs are also accompanied by not satisfied or poor health care results. The topic of this paper addresses an understudied and essential element that can improve the learning process by using Double-Loop Learning Theory (by Argyris \& Schon) in Purchasing process of the healthcare organizations, which will be added value to reduce the unnecessary costs especially that most of the public healthcare organizations are subject to shrinkage in their annual budget. This paper looks to the Essential cornerstone of learning in the process of decision making, and will examine how efficient and practical will be the strategies of behavior in this specialty involve share of organization power with any staff who has the skills and capabilities, and with any member of the organization who is competent in to be involved in decision making process or action implementation, in any defined task, or taking control of the environment within the organization. [Note: This is part of a larger thesis project: Learning Strategies in Healthcare Organizations in Kingdom of Saudi Arabia].
\end{abstract}

Article Information:

Received: $\quad 20^{\text {th }}$, April 2019

Revised: $3^{\text {rd }}$, Aug 2019

Accepted: $\quad 1^{\text {st }}$ Sep 2019

\section{Introduction}

\section{What is Learning?}

Learning is defined as setting learning goals, then monitor the progress, adjust any newly acquired cognition and behavior which will lead to gain the final goal of learning. The learner should be aware of his lack of knowledge and information, recognize the importance and urgency to learn, and must decide himself start the learning process (Jeong \& Frye, 2019). The world around us is very complex, with multi-dimensional nature that makes a big challenge for learning to see environment in a way that helps take the right skills of decision making in the different aspects of life. Humans learned from trial and errors and substantial progress has been made to reach the current huge knowledge. (Radulescu, Niv, \& Ballard, 2019).

The definition of learning is widely varied across disciplines, affected by different approaches used to assess its outcomes, as it difficult to reach common conceptualization of learning as different learning definitions used in different contexts. Learning is major focus of researches in behavioral ecology, neuroscience, psychology, theory of evolution, and with sciences related to computer, in addition of some different branches of sciences, so the definition of learning differ enormously between those different specialties, and everynow and then some new definitions are proposed by the researches based on the continous efforts of researches and development. Many debates happened about learning definition that increases the level of uncertainty about the borders of the concept of learning and the evaluation of its outcomes. The list of definitions of learning are different based on the field and disciplines, however the definition can be aligned under one broader concept of definition of learning which can be applied over all areas by using the definition of learning as general concept which is the process of getting information extracted from experiences aiming to build or develop system properties within the organization. We can also use the other and different definitions used by the individual sub-fields while at the same time added it to the common broader concept. While the specific satisfactory definition of learning can vary largely among fields and even with the same field, most recently theoretical approaches of learning definition is as structured methodology of updating of system properties of the organization based on processing of new information or knowledge (Barron et al., 2015).

\section{Why Learning is important?}


Society structure has been changed in the 21 st century due to the revolution in technology, facilitating mankind improvement in all aspects. Learning should be lifelong process should take place throughout life and in any situations. States and societies should adapt themselves and learn their people according to new era and changes in the world. (Ates \& Alsal, 2012). Learning is an important aspect to gain knowledge, which is the key success for the development of life of mankind. There is evidence that the prevailing environmental changes that can have impact on the different strategies of learning that can evolve and develop (Bullinaria, 2018). Learning is important especially implementing of evidence-based practices and interventions within child welfare as necessity. This requires a strong culture inside the organization that can emphasize and pushes to translate the evidencebased roles to daily practices and manners of the staff and part of learning operation, which will improve the adaptation skills of children. This should include clear communication practice, training and professional development of knowledge, value, and skills needed. (Julien-Chinn \& Lietz, 2019). Learning is important for individuals regardless they got it from experience and/or imitation. It helps the decision-makers process and its related information. (Offerman \& Sonnemans, 1998). As in this time of rapid changes, it become very important to rely on continuous learning as long-term strategy with the presence of challenges of shrinkages of budget, major steps in changes in different education level especially higher education and the continually increasing demand for resources and different services (McClamroch, Byrd, \& Sowell, 2001).

Learning also is important for transferring of knowledge to implement or doing the adaptation for future same situations to transfer the knowledge and experience. So, the transfer, then is a method to of perception, evaluation thinking and information processing, which is essential of all types of learning, and without this process we can not get involvement and engagement of our daily process of thinking and logical evaluation and even need the basic process of learning; transfer is playing the major role for the simplest ideas and the most complicated processes and achievements. We continually try to transfer our previous knowledge and experiences to get more satisfactory learning of new skills. The presence of computer makes it the fundamental toll of learning. The researches on teaching and learning show that the best ways which reinforce the knowledge and using different examples and concepts in multiple contexts of previous experiences of similar situations (Haskell, 2001). Learning also is important at workplace within the organization, as the new oversees competition of different products and services and accelerated innovations of technologies which lead to continuous unstable business environment with rapid changes. An essential part of this new business environment is creating increased demands on staff within the organization to get new knowledge and skills by the continuous learning process. So the learning is key for organizations to survive and to maintain market share with this tough oversees competition (Paulsson, Ivergård, \& Hunt, 2005).

\section{What is Organization learning?}

The definition of organizational learning can simply be known as learning by using the daily routines, depend on historical date of performance and this process should be target oriented. Accordingly, for the organization there should be referencing from the previous history of the organization to control the behavior. The organizational learning can be getting from direct day-to-day experience or getting from the experience from other organizations that can lead to building a model helping to interpret that experiences and out it in framework easy for learning. However the final and actual behavior that will be dominant within the organization is subjected to complicated bargaining process of different powers inside the organization to create the final learning model. We can understand the organizational learning by using three classical observations; First one, organization behavior that based routines are using policies and process to deal with situation more that selected choices. The second one the actions of the organization nare depend on history, as the routine-based work is usually based on understanding and interpretation of history rather than anticipations of future tasks. The third one is the organization is targetoriented, their behavior depends on how the outcome far or close the assigned targets. So, with such framework, the organization learning will happen by encoding the referencing from the organization history to be implemented to the routine work which will be as guidance of organizational behavior (Levitt \& March, 1988).

We can differentiate organizational learning from individual learning in methods such as; although the learning of organization individual is crucial and valuable for any organization, organizational learning is not about to add up the total of its individual learning, as organizations have its unique learning system which can affect their direct staff, also transmitted it to others by tracking its history, norms and behavior such as; (1) Organization culture; as the organization culture dominate itself in the overriding ideologies and start its own pattern of behavior. The organizational culture contains common values, ideologies, and beliefs that affect the decision making process. (2) Organizational strategy; the strategy in place is partially responsible for the capacity learning within organization. The main purpose of the strategy is to put organizational objectives and goals with clear pathway of to achieve that strategy, so it has high influence over learning process as the working environment is directly affected by the strategy. (3) Structure of the organization that helps the innovations and put in tract as essential part of organizational activities. (4) The environment within the organization, which can be internal or external factors and how the organization dealing with pressures and stress that can happen, which will affect the learning process, as the relationship between the learner and the environment should be stable and fit the learning process. (Fiol \& Lyles, 1985).

Organizational knowledge and faiths are transferred to the members via many forms of instructions, indoctrinations, and exemplification. An organization tries to emphasize and embedding the communications styles, values and processes that will build a strong organizational code. The organizational code is responsible to control the beliefs of the individual. This process will create mutual learning relationship between the organization and its individuals (March, 1991).

There are knowledge related constructs of organizational learning which will be described in (Figure 1):

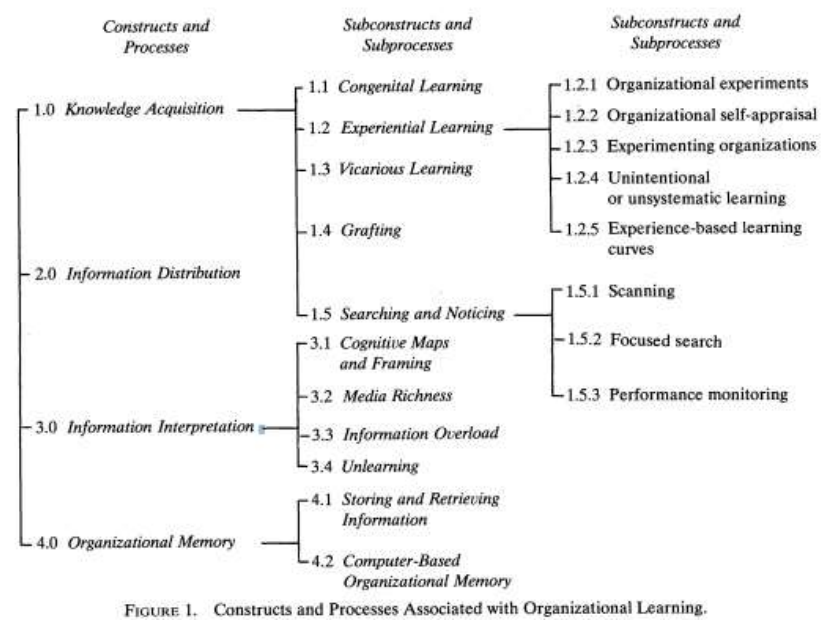

Fig.1: The Structure and process related to the organizational learning process in detailed methodology. 
If we take a look into the four learning-related constructs (See Fig 1), each will have subsequent action. The acquisition of knowledge, the process by which the organization can get knowledge from different resources. Distribution of the information, which is the process of sharing the information for better understanding. The interpretation of the information, based on common understanding. A memory of the organization, where the information and knowledge store for future process of organizational learning (Huber, 1991).

\section{Argyris \& Schon Single Loop and Double Loop Theories}

\section{The Essential Role of Learning in the Process of Decision Making}

The learning process can be defined as error detection and correction, as the errors are any defects that make actions ineffective. As the role of detecting and correcting the errors will provide the organization with learnings options and lack of it prevent learning. It very important to understand the nature and complexity of the process of decision making which consist of many steps and factors to make it succeeded. There two main factors that make the process of learning within the organization non-effective. The first is the level of interpersonal relationship within the organization, bureaucracy, and transparency of effective decision. The second is the feedback mechanism and corrective action based on it.

\section{Applied theories of action in organizational learning}

Argyris and Schon (1974) mentioned that actions done by human being should be related to or based on theories of action. We have two kinds of theories; the espoused one related to the theories used by individuals as the base of their action, while theories-in-use can be defined as the actual behaviors done by the individuals.

\section{SINGLE LOOP MODEL}

This methodology based on theory-in-use, it has hypothesis that any behavior in different situations is representing the best option in front of people facing that situation and matching their governing values and variables, such as achieving certain tasks or entering winning competition, dealing with hard feelings by emphasizing rationality. It also has another hypothesis that the learning process of human beings is linked with their strategies regarding the behavior associated with governing values and variables. The first strategy in use is to solve the issues is using unilaterally control to protect themselves and the group. The main behavioral strategy used here is to control others. Using a control strategy will influence leaders, other staff and the organizational environment, but it will produce disadvantages of defensiveness and closed culture, as the unilateral control prevent valid and neural feedback. Accordingly the solving of the problems of all issues will not be effective. We can refer to the single loop learning as the process of detection of errors then correct it to improve the performance of the organization but without touching or changing organizational values, norms or system. This kind of learning can be applied to the transactional problems within the organization.

\section{DOUBLE-LOOP MODEL}

The main reasons for using double-loop learning is to avoid the outcomes of the previous strategy of single-loop learning. In this model there will be changes in the values and variables of the organizations as it should be changed for the purpose of errors detection and correction. For example in the single-loop model considering that individuals will be linked to goals, purposes, and targets, and at the same time have control over others and the environment for the purpose to make sure about the achievement of their goals. While the double loop model, there will be no unilateral control, as the ultimate target is to get valid information as accurate as possible and to make the participant become internally committed. Accordingly, leaders should be well trained and skillful to apply to learn by double-loop learning model. All actions in this model are evaluated based on the level and degree that helps the participant to give valid and accurate information, which can include the feelings and emotions and solving problems effectively. The behavioral strategies applied here are sharing power with any individual who is competent to contribute effectively in decision making process to achieve defined tasks and have control of the organizational environment. With such conditions, individuals will not compete with others to push for their decisions for just selfgratification, rather they will be ready to look for to find the competent individuals to cooperate with for making the correct decisions, and maximizing the contribution of all individuals which will be reflected on teamwork and synergy within the organization (Argyris, 1976).

The process of solving the problems is the key task of any successful organization, but usually the individuals involved in this task facing issues to get proper and accurate understanding of the nature of errors, lack of concentration due to daily workload, which will lead to that the proposed solution with not be satisfactory. The problemsolvers need to be expert in hard and soft knowledge, with proper skills and capabilities to enabling them to identify the root cause of the errors then they will $\mathrm{b}$ able to fix it (Al-Raqadi, Abdul Rahim, Masrom, \& AlRiyami, 2016). This model used when the individuals within the organization try to challenge the existing organizational values and norms that were in use, this step aiming at developing new and innovative ways for errors detection and fixing. Therefore it will involve and engages all individuals within the organization to provide the necessary accurate information, by asking questions about the root cause of the problem, for fixing and preventing in future similar situations. This provides strong proof that double-loop learning is helping as catalyst for creating knowledge in organizations (Jaaron \& Backhouse, 2017).

\section{Problem statement}

Healthcare organizations across the world begun to prioritize the procurement of sustainability-produced materials. This due to the constraints resulted from issues in economy and logistic support in healthcare organizations related to purchasing process to provide medications, foods, medical consumables and materials to the patients, many new trials from health care organizations are struggling in matching with the traditional system of supply chain and its norms, standardizations, and reliability during the process of trying to meet other criteria for example mutual benefits within supply chain processes, environmental factors, and limitation of healthcare budget.(Klein, 2015). The healthcare organization and its financing have been shifted to totally changing in past decade. As transformation process of public healthcare organizations to have control over the purchasing process to control the cost of the supply chain management. The new changes have big impact on healthcare consumers and the delivery of healthcare products and services. There are three key factors affecting this process. The first one is the sponsoring role; which including eligibility process of getting health care services, accountability to make sure that the consumers receiving the services under the agreed scope of benefits. The second one the purchasing and payment roles and regulations, the filtration process to control the utilization of products and services including triaging. The Third factor is providing behavioral healthcare services to the eligible members that 
have access to healthcare system (Rosenthal, Minden, Manderscheid, \& Henderson, 2006). In USA, the annual spending of healthcare per person is around $\$ 8,300$, compared to Norway and Switzerland in spending per person which located as second (Zamosky, 2013). If we take a look to the countries that have a big and expanded healthcare system, we can the medications and pharmacy products are representing the major part of total healthcare expenditure around $60 \%$ and most of those counties are middle-income countries. This makes pressures on most of the countries and make them strive toward to collected or universal healthcare coverage, to reach pocket spenders on private sections, but this will cause increased expenditures and put extra budget on public section. This will have bad impact on service level provided (Maniadakis, Holtorf, Otávio Corrêa, Gialama, \& Wijaya, 2018). There is significant relationship between national expenditure on healthcare and gross domestic product (GDP) (Maniadakis et al., 2018). If we take Saudi Arabia as example, we can see that the healthcare budgeting is increasing annually while the national income decreasing which is making huge burden (Fig 2). Through KSA the new innovative program for the national economic transformation plan of the Saudi economy called National Transformation Program 2020, as trial to do major changes in the national economy from oil-dependant to diversified econom. This program have special care of healthcare system aiming to acchieve best care quality and effectiveness of service delivery. Considering the financial constraints due to decreased oil prices which is the main source of Saudi economy. Bringing innovations and change to healthcare system is a major milestone due to the complexity in its structure and functions. This will lead to introduce changes in governance of healthcare authorities and maanagement to all kinds of healthcare organizations, small or large, public or private due to the diversity of issues in both technical and human complexities

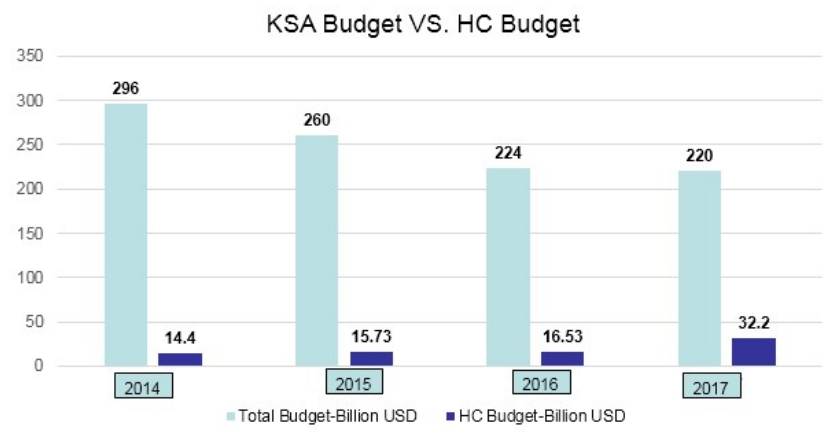

Fig. 2: KSA Budget VS. HC Budget

\section{Research Questions and Objectives}

We will try to answer the below question:

1-What are the most important opportunities of the healthcare organization to implement Double-loop Model?

2-How Double-loop model Transformation could affect a better financial performance in the purchasing process of healthcare organization?

3-Why learning is important in a healthcare organization?

So, the objectives of this paper are reviewing the relationships of the existing setup, in order to attain the following:

1-To explore the opportunities of the healthcare organization to implement Double-loop Model in the purchasing process.

2-To evaluate the impact especially financially of the implementation of Double-Loop Model toward cutting the unnecessary cost.
Operational Definitions

\section{Healthcare}

Healthcare can be seen as a service. As there are no difference between, healthcare or healthcare services. Vargo and Lusch's (2004) definition of service as the utilization of specific competencies either skills or knowledge through actions, processes, and performance for the advantages of another entity or the entity itself. It has a variety of items compared to other kinds of services, which includes patients' vulnerable position that should be considered a special case (Berry and Bendapudi, 2007).

\section{Healthcare Organization}

It can be defined as the organization that supplying and providing health services and have nurses, doctors, pharmacist, etc. The main role of a healthcare organization is providing well-being services with reasonable prices and maximum availability of services upon order. The main goal of HCOs is treating of patients maintaining the highest standards although increased demands on healthcare services. (Rodríguez Perera \& Peiró, 2012).

\section{Service Innovation in Healthcare}

It can be defined as per the dictionary to make new amendments or changes in something already exist or established either new methods or ideas or products and services (Oxford Dictionary, 2013). There are multiple points of view presented by the researchers, most importantly the ones related to innovations of disease prevention and providing access to healthcare services especially the one provided by Djellal and Gallouj (2007). When used in this thesis service development refers to planned service innovation processes.

\section{Literature Review}

\section{Organizational learning}

So many reviews about the organizational learning literature, that shows a different perspective on types and level of required learning and trying to provide clear explanations about the relationship between learning and performance. Published organizational learning researches have more focus on the process of decision making and choice. There is also conceptual confusion about organizational learning with knowledge management and intellectual capital. (Bontis, Crossan, \& Hulland, 2002). There are three levels of organizational learning in a healthcare organization.

\section{Individual-level learning:}

It can be defined as the steps of developing new insight into the individual. This process will utilize the tacit knowledge and expertise. The new insights should be translated into a new process. We should also emphasize on putting criteria to make sure that all individual has proper understanding, so they can have a cognitive map generated from 
interpreting. This cognitive maps will be as a stock of individual learning, knowledge, and competencies. These competencies can be specified to a job context. (Argyris, 1976).

\section{Group level Learning:}

The more emergent nature of learning by individual will generate ongoing interactions within the organization members. Group learning will involve the utilization of staff interpretations on an individual level to develop a common understanding of a specific case. It will happen through the continuous conversations among the individuals of the organization sharing the understanding. We use the term group rather than the commonly used term; team learning because sometimes there is no specific team but an only group of individuals. (Marsick \& Watkins, 1994). It also includes teamwork, productive meeting, diversity, and problem-solving dialogue.

\section{Organization level of learning:}

So many different views about the organization learning, some make it related to the collection of individual (human perspective), others look to it as system, policy, procedures, and structure (nonhuman perspective) that constitute the history of the organization. (Fiol \& Lyles, 1985). The organizational learning is bigger than the meaning of just shared information. It representing the translation of the understanding of new products, processes, services, strategy, and structure. (Crossan \& Berdrow, 2003). So the organization level of learning involves embedding individuals and group learning into the non-human aspects within the organization including system, strategy, structure, and procedure. (Walsh \& Ungson, 1991).

\section{Argyris \& Schon Double-Loop Model in Purchasing Process of Healthcare Organization}

In Double-Loop learning, every single action is calculated based on the level that can contribute to fix the problem or toward a better and deep understanding of the issues, as the unilateral control by the individuals that usually associated with advocacy is rejected. Accordingly, the advocacy is usually accompanied with confrontation one to another view to and to alter them in order to have the position that based on the most detailed use of information, so the individuals are internally committed. (Reychav, Kumi, Sabherwal, \& Azuri, 2016). Double-Loop learning model happens when errors are rectified changing the governing values and norms before doing the necessary corrective actions. (Henderson, Blackman, \& Connelly, 2004). DoubleLoop Learning (DLL) consider as the core concept and includes the idea that an organization continuously learns and innovate as grouped experiences, for example, all success and failures are learning opportunities will be used as a reference framework for continuous corrections on organization governing values and variables. (Matthies \& Coners, 2018), so this means that all learned situations from past experiences are not only used for future errors-fixing in same situation but for also re-evaluation and amendment of the governing values and standards of the organization using the knowledge capital generated.

Transferred this model to purchasing process in a healthcare organization, DLL means that lessons learned from past processes happened in order to develop a framework for the design and implementation of future processes. (Brady \& Davies, 2004). An important source of knowledge of such learning is the documentation of whole process in details of previously purchased items done before, which will make such knowledge available for reuse I future. So the ultimate target of this process organizational learning is to develop organizational skills, which will be based on the critical exploration of learned lessons, for example solved problems and subsequent changes in behavior, therefore the concept of reflective learning that not only used for isolated problem solving, but to also examine and modify the organizational standards, policy and procedures, aiming to continuous and profound changes in high-level governing values and variables of the whole organization (Argyris, 1976).

In the literature, there is some proposed implementation of DLL concept, but we will concentrate on the implementation on an organizational level, as which promote collective Double-Loop learning (DLL) through organizational structure, policy, and process (Brady \& Davies, 2004). It also suggested building learning alliances to foster DLL through the exchange of knowledge of interorganizational partnership, which will support decision-making in specific learning conditions and accordingly DLL facilitate the processes in the organization. (Walker \& Christenson, 2005), as illustrated in (Fig. 3):

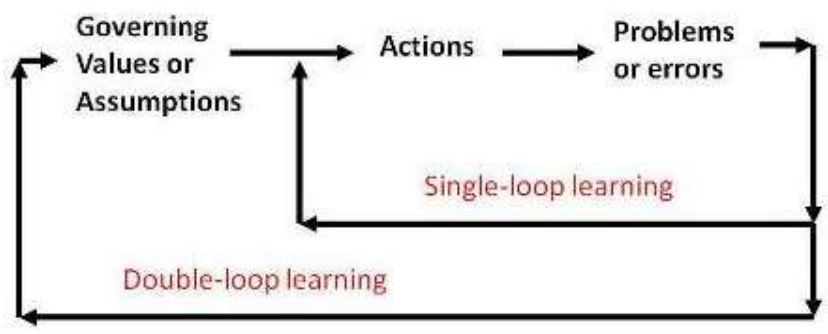

Argyris and Schon, 1978

Fig. 3: Conceptual Framework of Double-Loop Learning

The core function of the implementation of Double-Loop Learning (DLL) in purchasing process in healthcare organization aims to evaluate the future process based on historical lessons, which means that the concept of new process should be the ability to implement the lessons collected from previous historical process, which will help to come up with innovations in healthcare sector (Matthies \& Coners, 2018).

There will be some limitations such as the content of corresponding experience reports will have much missing information and maybe will not be trustworthy, so the data cleaning and accuracy should be the top priority (Cheng, Schulz, \& Booth, 2009).

This innovative process expected to have some reflections of the performance of healthcare organization, such as allowing correction of distortions and errors in beliefs in the organization, allow the identification and resolution of practical problems through rationalization, enhance self-esteem through learning, lead to acceptance of professional responsibility, and allow the generation of knowledge-based on more comprehensive learning from past lessons (Greenwood, 1998).

\section{Sustainability of Healthcare Organization based on Learning}

Few publications have reported the sustainability of healthcare improvement. Sustainability is a key implementation outcome on scientific basis (Proctor et al., 2015). It is obvious that the important tool of the organization to do a transition to innovative models is by organizational learning structures and processes (Dicle \& Köse, 2014). The main purpose of organization learning toward the sustainability plan is to increase the efficiency of the organization by acquiring and organizing knowledge to increase the skills of its organizational 
members, which means the process in which the organizations draw lessons from history, interpret and put them into organizational routine. The organizational environment can have competitive advantages by providing strategic capabilities such as continuous innovations, control compliance, and high-order learning (Levitt \& March, 1988).

\section{Expected Findings}

We are expecting to prove that the holistic concept of learning will not be limited to help to achieve higher productivity and efficiency, it also will help individuals to understand and be aware of other important non-tangible values; such as moral and ethics, which play an important rule in any organization in term of continuous improvement.

Moreover, using two-ways dialog and regular amendments on organizational governing values and variables, for proper utilization of the knowledge and information, for continuous problem solving and innovations in healthcare organizations. (Othman, 2014)

This paper part of my larger thesis project (in progress) as a qualitative study to prepare for grounded theory: Learning Strategies in Healthcare Organizations in Kingdom of Saudi Arabia. From literature, it shows that Double-Loop Learning can be implemented on healthcare organization which will lead to organizing the acquired knowledge which is utilized to create competitive advantages and implement innovations derived from lessons from history and put them into an organizational routine.

Healthcare purchasing process, Double-Loop Learning (DLL) will be an essential part of the sustainability plan to increase the efficiency of all process which will be reflected in financial results of the organization. It also allowing correction of distortions and errors in beliefs in the organization, allow the identification and resolution of practical problems through rationalization, enhance self-esteem through learning, leads to acceptance of professional responsibility, and allow the generation of knowledge-based on more comprehensive learning from past lessons.

\section{References}

Al-Raqadi, A. M. S., Abdul Rahim, A., Masrom, M., \& Al-Riyami, B. S. N. (2016). System thinking in single- and double-loop learning on the perceptions of improving ships' repair performance. International Journal of System Assurance Engineering and Management, 7(1), 126-142. doi:10.1007/s13198-015-0353-7

Argyris, C. (1976). Single-Loop and Double-Loop Models in Research on Decision Making. Administrative Science Quarterly, 21(3), 363-375. doi: $10.2307 / 2391848$

Ates, H., \& Alsal, K. (2012). The Importance of Lifelong Learning has been Increasing. Procedia - Social and Behavioral Sciences, 46, 40924096. doi:https://doi.org/10.1016/j.sbspro.2012.06.205

Barron, A. B., Hebets, E. A., Cleland, T. A., Fitzpatrick, C. L., Hauber, M. E., \& Stevens, J. R. (2015). Embracing multiple definitions of learning. Trends in Neurosciences, 38(7), 405-407. doi:https://doi.org/10.1016/j.tins.2015.04.008

Bontis, N., Crossan, M. M., \& Hulland, J. (2002). Managing An Organizational Learning System By Aligning Stocks and Flows. Journal of Management Studies, 39(4), 437-469. doi:10.1111/1467-6486.t011-00299

Brady, T., \& Davies, A. (2004). Building Project Capabilities: From Exploratory to Exploitative Learning. Organization Studies, 25(9), 1601-1621. doi:10.1177/0170840604048002

Bullinaria, J. A. (2018). Evolution of learning strategies in changing environments. Cognitive Systems Research, 52, 429-449. doi:https://doi.org/10.1016/j.cogsys.2018.07.024

Cheng, M. M., Schulz, A. K. D., \& Booth, P. (2009). Knowledge transfer in project reviews: the effect of self-justification bias and moral hazard.
Accounting \& Finance, 49(1), 75-93. doi:10.1111/j.1467629X.2008.00271.x

Crossan, M. M., \& Berdrow, I. (2003). Organizational learning and strategic renewal. Strategic Management Journal, 24(11), 1087-1105. doi:10.1002/smj.342

Dicle, Ü., \& Köse, C. (2014). The Impact of Organizational Learning on Corporate Sustainability and Strategy Formulation with the Moderating Effect of Industry Type. Procedia - Social and Behavioral Sciences, $\quad 150, \quad 958-967$. doi:https://doi.org/10.1016/j.sbspro.2014.09.108

Fiol, C. M., \& Lyles, M. A. (1985). Organizational Learning. Academy of Management Review, 10(4), 803-813. doi:10.5465/amr.1985.4279103

Greenwood, J. (1998). The role of reflection in single and double loop learning. Journal of Advanced Nursing, 27(5), 1048-1053. doi:10.1046/j.1365-2648.1998.00579.x

Haskell, R. E. (2001). Chapter 2 - Transfer of Learning: What It Is and Why It's Important. In R. E. Haskell (Ed.), Transfer of Learning (pp. 23-39) San Diego: Academic Press.

Henderson, S., Blackman, D., \& Connelly, J. (2004). Does double loop learning create reliable knowledge? The Learning Organization, 11(1), 1127. doi:10.1108/09696470410515706

Huber, G. P. (1991). Organizational Learning: The Contributing Processes and the Literatures. Organization Science, 2(1), 88-115. doi:10.1287/orsc. 2.1 .88

Jaaron, A. A. M., \& Backhouse, C. J. (2017). Operationalising “Double-Loop" Learning in Service Organisations: A Systems Approach for Creating Knowledge. Systemic Practice and Action Research, 30(4), 317-337. doi:10.1007/s11213-016-9397-0

Jeong, J., \& Frye, D. (2019). Self-regulated learning: Is understanding learning a first step? Early Childhood Research Quarterly. doi:https://doi.org/10.1016/j.ecresq.2018.12.007

Julien-Chinn, F. J., \& Lietz, C. A. (2019). Building learning cultures in the child welfare workforce. Children and Youth Services Review, 99, 360365. doi:https://doi.org/10.1016/j.childyouth.2019.01.023

Klein, K. (2015). Values-based food procurement in hospitals: the role of health care group purchasing organizations. Agriculture and Human Values, 32(4), 635-648. doi:10.1007/s10460-015-9586-y

Levitt, B., \& March, J. G. (1988). Organizational Learning. Annual Review of Sociology, 14(1), 319-338. doi:10.1146/annurev.so.14.080188.001535

Maniadakis, N., Holtorf, A.-P., Otávio Corrêa, J., Gialama, F., \& Wijaya, K. (2018). Shaping Pharmaceutical Tenders for Effectiveness and Sustainability in Countries with Expanding Healthcare Coverage. Applied Health Economics and Health Policy, 16(5), 591-607. doi:10.1007/s40258-018-0405-7

March, J. G. (1991). Exploration and Exploitation in Organizational Learning. Organization Science, 2(1), 71-87. doi:10.1287/orsc.2.1.71

Marsick, V. J., \& Watkins, K. E. (1994). The learning organization: An integrative vision for HRD. Human Resource Development Quarterly, 5(4), 353-360. doi:10.1002/hrdq.3920050406

Matthies, B., \& Coners, A. (2018). Double-loop learning in project environments: An implementation approach. Expert Systems with Applications, $\quad 96, \quad 330-346$. doi:https://doi.org/10.1016/j.eswa.2017.12.012

McClamroch, J., Byrd, J. J., \& Sowell, S. L. (2001). Strategic Planning: Politics, Leadership, and Learning. The Journal of Academic Librarianship 27(5), 372-378. doi:https://doi.org/10.1016/S0099-1333(01)00222-

Offerman, $\bar{T}$., \& Sonnemans, J. (1998). Learning by experience and learning by imitating successful others. Journal of Economic Behavior \& Organization, 34(4), 559-575. doi:https://doi.org/10.1016/S01672681(97)00109-1

Othman, M. F. (2014). Application of the Learning Organization in a Muslim Non Governmental Organization. Universiti Teknologi Malaysia,

Paulsson, K., Ivergård, T., \& Hunt, B. (2005). Learning at work: competence development or competence-stress. Applied Ergonomics, 36(2), 135 144. doi:https://doi.org/10.1016/j.apergo.2004.09.008

Proctor, E., Luke, D., Calhoun, A., McMillen, C., Brownson, R., McCrary, S. \& Padek, M. (2015). Sustainability of evidence-based healthcare: research agenda, methodological advances, and infrastructure support. Implementation Science, 10(1), 88. doi:10.1186/s13012015-0274-5 
Radulescu, A., Niv, Y., \& Ballard, I. (2019). Holistic Reinforcement Learning: The Role of Structure and Attention. Trends in Cognitive Sciences, 23(4), 278-292. doi:https://doi.org/10.1016/j.tics.2019.01.010

Reychav, I., Kumi, R., Sabherwal, R., \& Azuri, J. (2016). Using tablets in medical consultations: Single loop and double loop learning processes. Computers in Human Behavior, 61, 415-426. doi:https://doi.org/10.1016/j.chb.2016.03.020

Rodríguez Perera, F. d. P., \& Peiró, M. (2012). Strategic Planning in Healthcare Organizations. Revista Española de Cardiología (English Edition), 65(8), 749-754. doi:https://doi.org/10.1016/j.rec.2012.04.004

Rosenthal, M. B., Minden, S., Manderscheid, R., \& Henderson, M. (2006). A Typology of Organizational and Contractual Arrangements for Purchasing and Delivery of Behavioral Health Care. Administration and Policy in Mental Health and Mental Health Services Research, 33(4), 461-469. doi:10.1007/s10488-005-0025-z

Walker, D. H. T., \& Christenson, D. (2005). Knowledge wisdom and networks: a project management centre of excellence example. The Learning Organization, 12(3), 275-291. doi:10.1108/09696470510592520

Walsh, J. P., \& Ungson, G. R. (1991). ORGANIZATIONAL MEMORY. Academy of Management Review, 16(1), 57-91. doi:10.5465/amr.1991.4278992

Zamosky, L. (2013). Cutting Medical Costs. In L. Zamosky (Ed.), Healthcare, Insurance, and You: The Savvy Consumer's Guide (pp. 75-88). Berkeley, CA: Apress. 